\title{
Factores asociados a mejoría visual a corto plazo tras implantación de segmentos intraestromales en pacientes con queratocono
}

\section{Factors associated with short-term visual improvement after intracorneal ring segments implantation}

\author{
Ernesto Soto-Masías ${ }^{1 *}$, Tomas Galvez-Olortegui ${ }^{2,3}$, Jose Galvez-Olortegui ${ }^{3,4,5,6}$, Fernando lyo-Alberti ${ }^{7}$ \\ Gladys Delgado-Becerra ${ }^{1}$
}

${ }^{1}$ Servicio de Enfermedades Externas, Córnea y Cirugía Refractiva, Instituto Nacional de Oftalmología Dr. Francisco Contreras Campos, Lima; ${ }^{2}$ Departamento de Oftalmología, Hospital Nacional Guillermo Almenara Yrigoyen, Lima; ${ }^{3}$ Unidad de Oftalmología Basada en Evidencias (Oftalmoevidencia), Scientia Clinical and Epidemiological Research Institute, Trujillo; ${ }^{4}$ Vicerrectorado de Investigación, Universidad Nacional Santiago Antúnez de Mayolo, Huaraz; ${ }^{5}$ Dirección ejecutiva de Investigación y docencia especializada en oftalmología y desarrollo de tecnologías, Instituto Nacional de Oftalmología, Lima; ${ }^{6}$ Unidad de Investigación Clínica, Scientia Clinical and Epidemiological Research Institute, Trujillo; ${ }^{7}$ Servicio de Consultorios Generales, Instituto Nacional de Oftalmología Dr. Francisco Contreras Campos, Lima. Perú

\section{Resumen}

Objetivo: Determinar la asociación entre factores preoperatorios y la mejoría visual a corto plazo después del implante de segmentos intraestromales en pacientes con queratocono, así como conocer sus complicaciones operatorias. Método: Estudio observacional, analítico de casos y controles. Se incluyeron 110 ojos de 91 pacientes. Los factores preoperatorios estudiados fueron edad, sexo, agudeza visual preoperatoria, refracción, técnica quirúrgica empleada (mecánica o asistida por femtosegundo), paquimetría y grado de queratocono según queratometría. Se formaron dos grupos según la ganancia de líneas de visión sin correctores después de la cirugía de implante de segmentos intraestromales, con ganancia mayor o igual a tres líneas (casos) y ganancia menor a tres líneas (controles). Resultados: De las variables estudiadas, solo el estado refractivo previo de astigmatismo miópico tuvo una frecuencia mayor estadísticamente significativa $(p<0,05)$ en el grupo de casos que en el de controles. Aunque en el grupo de casos se encontró mayor número de ojos con queratometrías medias entre 48 y $52 D$ (grado II de queratocono), este valor no resultó estadísticamente significativo. No hubo asociación entre la técnica operatoria utilizada y el resultado visual postoperatorio. El porcentaje de complicaciones operatorias fue del 1.81\%. Conclusiones: El estado refractivo previo fue el único factor estudiado con una asociación estadísticamente significativa con la agudeza visual sin correctores postoperatoria. La presencia de complicaciones postoperatorias fue baja.

Palabras clave: Queratocono. Segmentos intraestromales. Topografía corneal. Ectasia corneal. Cirugía de queratocono. Terapia de queratocono.

\section{Abstract}

Objective: To evaluate the correlation between preoperative factors and short-term postoperative visual improvement in patients with keratoconus treated with intracorneal ring segments implantation. Additionally, to determine surgical complications.

Correspondencia:

*Ernesto Soto-Masías

Avenida Tingo María, 398

Cercado de Lima

Fecha de recepción: 07-12-2019

C.P. 15082, Lima, Perú

E-mail: sotomasias@ hotmail.com DOI: 10.24875/RMO.M20000119

0187-4519/○ 2020 Sociedad Mexicana de Oftalmología. Publicado por Permanyer. Este es un artículo open access bajo la licencia CC BY-NC-ND (http://creativecommons.org/licenses/by-nc-nd/4.0/).
Disponible en internet: 01-07-2020 Rev Mex Oftalmol. 2020;94(4):159-165

www.rmo.com.mx 
Methods: Analytical, observational case-control study. One-hundred and ten eyes of 91 patients were included. The preoperative factors evaluated included age, sex, visual acuity, refractive error, surgical technique (mechanical or femtosecond-assisted), pachymetry and keratoconus grade by keratometry. Two groups were obtained according to the gain of lines of uncorrected visual acuity after intrastromal segment implantation surgery, with a gain of 3 or more lines (cases), and a gain of 3 or less lines (controls). Results: Only preoperative myopic astigmatism had a statistically significant higher frequency in cases than in controls $(p<0.05)$. Although there was a greater number of eyes with an average keratometry between 48 - $52 D$ (Keratoconus stage II) in the cases group, this was not statistically significant. There was no correlation between the preoperative technique used and the postoperative visual result. The percentage of surgical complications was of $1.81 \%$. Conclusion: The only factor with a statistically significant correlation with uncorrected visual acuity was the previous refractive error. The incidence of postoperative complications was low.

Key words: Keratoconus. Intracorneal ring segments. Corneal topography. Corneal ectasia. Keratoconus surgery. Keratoconus therapy.

\section{Introducción}

El queratocono es una enfermedad degenerativa progresiva no inflamatoria bilateral, que puede presentarse como adelgazamiento del estroma corneal; la mayoría de veces es asimétrica, y su etiología aún es desconocida'. El inicio de los síntomas puede ocurrir durante la adolescencia y la edad adulta joven, y puede manifestarse como visión reducida, astigmatismo corneal y visión fluctuante, lo que repercute negativamente en la calidad de vida de los pacientes ${ }^{1,2}$. Actualmente, el diagnóstico se ha vuelto cada vez más frecuente con el surgimiento de la cirugía refractiva y la topografía que se realiza preoperatoriamente para detectar cualquier cambio ectásico, así sea temprano ${ }^{3-5}$.

El objetivo principal del tratamiento es mejorar la visión y/o prevenir la ceguera ${ }^{1}$. El tipo de tratamiento depende de la gravedad, ya que inicialmente es médico, con anteojos o lentes de contacto rígidos permeables al gas ${ }^{1}$. Cuando estos métodos fallan, está indicada la cirugía para mejorar la superficie corneal. Cuando la córnea se mantiene transparente, la implantación de segmentos de anillos intrastromales de polimetilmetacrilato (PMMA) puede ser una opción exitosa ${ }^{6}$.

El objetivo de este estudio fue evaluar los posibles factores predictivos (del paciente o de la técnica quirúrgica) de un buen resultado visual sin correctores postimplantación de segmentos intraestromales corneales en pacientes con queratocono, y describir las complicaciones quirúrgicas.

\section{Materiales y métodos}

Se realizó un estudio de casos y controles. No se calculó muestra, se estudiaron los registros clínicos preoperatorios de la totalidad de pacientes con queratocono tratados con implante de segmentos intraestromales en el Instituto Nacional de Oftalmología (INO) Dr. Francisco Contreras Campos de Lima, Perú, durante 2018. Fueron 120 pacientes operados en ese lapso, de los cuales 93 fueron incluidos en el estudio por tener los criterios adecuados de selección en su registro médico. El queratocono fue diagnosticado por médicos especialistas del departamento de córnea, con base en la examinación clínica por biomicroscopia (visualización de signos como estrías de Vogt, anillo de Fleischer o adelgazamiento corneal), refracción (astigmatismos altos que no corregían totalmente), valores queratométricos superiores a $47 \mathrm{D}$ y estudio topográfico con paquimetría corneal menor a 500 um, e incremento de las elevaciones anteriores y posteriores corneales. Se dividió a los participantes en dos grupos (casos y controles) de acuerdo a la ganancia de líneas después de al menos 3 meses de la implantación de segmentos intraestromales. Se consideró buena ganancia cuando fue mayor o igual a 3 líneas (casos). Los criterios de exclusión para ambos grupos fueron una agudeza visual (AV) preoperatoria no corregida mejor a 0.3 LogMAR, otro tipo de ectasia corneal diferente a queratocono (degeneración marginal pelúcida o ectasia posterior a cirugía refractiva), implantación de segmentos de espesor variable, glaucoma, antecedente de cross-linking corneal u otra cirugía ocular previa. Se eliminaron las historias clínicas de pacientes con datos incompletos.

Los factores preoperatorios estudiados fueron: edad, sexo, AV preoperatoria (corregida, no corregida y las líneas de visión que ganaban con correctores), refracción, técnica quirúrgica empleada (mecánica o asistida por femtosegundo), paquimetría y grado de queratocono según queratometría. La AV no corregida y corregida se midió en notación de Snellen decimal y se convirtió al logaritmo del mínimo ángulo de resolución (LogMAR) para el análisis estadístico. Las variables queratometría y paquimetría se obtuvieron de la 
topografía Galilei G6 (Ziemer, Port, Suiza). Para la paquimetría se consideró el punto más delgado corneal en micras. El grado de queratocono en nuestro estudio se determinó según el valor de queratometría media $(\mathrm{km})$ de la clasificación de Amsler-Krumeich: grado I (< 48 D), grado II (48-52 D), grado III (53-55 D) y grado IV $(>55 D)^{7}$. Adicionalmente, se evaluaron las complicaciones intra y postoperatorias.

Todos los segmentos implantados fueron de PMMA de $5 \mathrm{~mm}$ de diámetro. Se colocaron uno o dos segmentos en cada ojo, con un espesor que iba de $150 \mathrm{a}$ 300 um (con pasos de 50 um) y longitudes de arco de $90^{\circ}, 120^{\circ}, 160^{\circ}$ y $210^{\circ}$. Se utilizaron segmentos de dos marcas diferentes: los Intraseg (Gamma Vision, Buenos Aires, Argentina) se implantaron en el $94.6 \%$ de casos y los Keraring (Mediphacos, Belo Horizonte, Brasil), en el $2.7 \%$. En un $2.7 \%$ de los pacientes se implantó uno de cada marca en el mismo ojo.

Para calcular el número de segmentos a implantar, así como su longitud y espesor, se consideró el nomograma de Mediphacos 2009. Los segmentos fueron implantados por cirujanos expertos. En la técnica manual se utilizó un cuchillete de diamante para la incisión, así como disectores semicirculares para la tunelización. En la técnica asistida por femtosegundo se utilizó el láser (Ziemer FEMTO LDV Z8) para crear los túneles y la incisión. En ambas, la incisión se realizó en el meridiano más curvo, y la profundidad de implantación a la que se apuntó fue del $80 \%$ en el estroma.

Los datos se analizaron en el software SPSS 24. Se utilizaron las pruebas $\mathrm{T}$ de Student, $\mathrm{U}$ de Mann-Whitney y Chi-cuadrado, y se consideró significativo a un valor de $p$ inferior a 0.05 . El estadístico de asociación utilizado fue el odds ratio (OR) para variables cualitativas, y para variables cuantitativas este se halló mediante el análisis de regresión logística, con un intervalo de confianza al $95 \%$.

La realización del presente estudio fue aprobada por el Comité de Ética del INO Dr. Francisco Contreras Campos. Todos los pasos de este estudio siguieron los principios de la Declaración de Helsinki. No se pidió consentimiento informado, ya que se utilizó una base de datos secundaria. Los datos obtenidos se utilizaron manteniendo la confidencialidad e intimidad de las personas que participaron en la investigación.

\section{Resultados}

Se incluyeron 110 ojos (54 casos y 56 controles) de 91 pacientes, de los cuales, 67 (73.6\%) fueron
Tabla 1. Características clínico-epidemiológicas de los pacientes con queratocono

\begin{tabular}{|c|c|c|}
\hline Característica & Casos & Controles \\
\hline Edad (media $\pm \mathrm{DE})$ & $22.13 \pm 10.3$ & $23.11 \pm 12.2$ \\
\hline $\begin{array}{c}\text { Sexo, n }(\%) \\
\text { Masculino } \\
\text { Femenino }\end{array}$ & $\begin{array}{l}40(74.1) \\
14(25.9)\end{array}$ & $\begin{array}{l}42(75) \\
14(25)\end{array}$ \\
\hline $\begin{array}{l}\text { Lateralidad, n (\%) } \\
\text { Ojo derecho } \\
\text { Ojo izquierdo }\end{array}$ & $\begin{array}{l}25(42.37) \\
29(56.86)\end{array}$ & $\begin{array}{l}34(57.63) \\
22(43.14)\end{array}$ \\
\hline
\end{tabular}

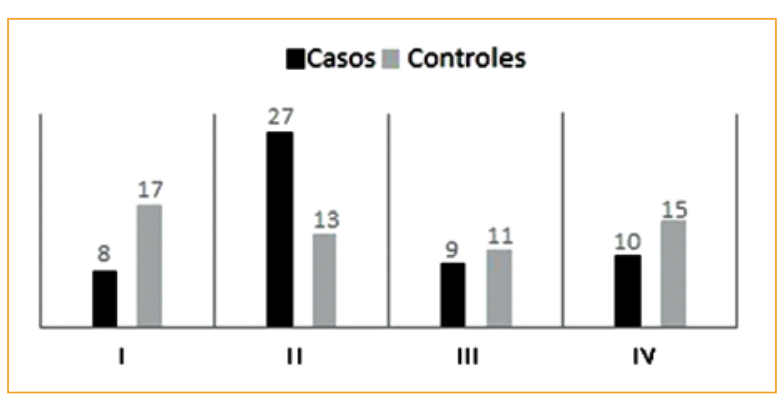

Figura 1. Número de pacientes en cada grupo según grado de queratocono.

hombres. La edad promedio fue $22.63 \pm 11.27$, con un rango de edades desde los 6 a los 75 años. Las características de la población de ojos incluidos en cada grupo de estudio se presentan en la tabla 1. Aproximadamente el $50 \%$ de los pacientes operados de segmentos intraestromales en el instituto procedían del departamento de Lima, mientras que un grupo importante pertenecía a la Sierra Central (19.8\%).

Dentro los resultados postoperatorios, el tiempo promedio transcurrido de la cirugía al control postoperatorio fue de 5.01 meses para los casos y de 4.3 meses para los controles. El $80 \%$ de los pacientes ganó al menos una línea de visión sin correctores después de la cirugía (61.8\% ganó dos o más). El 10.9\% se mantuvieron sin cambios, y solo el $8.2 \%$ empeoraron su visión preoperatoria sin correctores. No hubo diferencias entre ambos grupos en las AV preoperatorias (con y sin correctores) ni tampoco en relación con las líneas que se ganaban con correctores preoperatoriamente (Tabla 2).

Según la queratometría media, en el grupo de casos hubo un mayor porcentaje de pacientes con grado II (queratometrías medias entre 48 y $52 \mathrm{D}$ ) en relación con el grupo de controles (Fig. 1), pero al estudiar la asociación entre las variables y el resultado visual postquirúrgico mediante análisis de regresión logística, se encontró 
Tabla 2. Variables de estudio según los grupos estudiados

\begin{tabular}{|c|c|c|c|c|}
\hline Factor estudiado & Casos & Controles & Total & p \\
\hline AVSC preoperatoria (logMAR), mediana (RIQ) & $1.00(0.8-1.3)$ & $0.8(0.7-1.3)$ & $1.0(0.7-1.3)$ & $0.82^{*}$ \\
\hline AVCC preoperatoria (logMAR), mediana (RIQ) & $0.5(0.4-0.7)$ & $0.5(0.3-0.7)$ & $0.5(0.3-0.7)$ & $0.77^{*}$ \\
\hline Líneas de visión que ganaba con correctores preoperatorio, mediana (RI0) & $3.0(2.0-6.0)$ & $3.0(2.0-4.5)$ & $3.0(2.0-5.0)$ & $0.15^{*}$ \\
\hline $\begin{array}{l}\text { Grado de queratocono por queratometría, n (\%) } \\
\text { I y II }(\mathrm{Km}<53 \mathrm{D}) \\
\text { III y IV }(\mathrm{Km} \geq 53 \mathrm{D})\end{array}$ & $\begin{array}{l}35(64.8 \%) \\
19(35.2 \%)\end{array}$ & $\begin{array}{l}30(53.6 \%) \\
26(46.4 \%)\end{array}$ & $\begin{array}{l}65(59.1 \%) \\
45(40.9 \%)\end{array}$ & $0.23^{* *}$ \\
\hline Paquimetría preoperatoria (micras), media \pm SD & $434.22 \pm 36.63$ & $441.07 \pm 57.66$ & $437.71 \pm 22.63$ & $0.46^{* * *}$ \\
\hline $\begin{array}{l}\text { Defecto refractivo previo, } \mathrm{n}(\%) \\
\text { Astigmatismo miópico } \\
\text { Astigmatismo simple o } \\
\text { Hipermetrópico }\end{array}$ & $\begin{array}{c}50(92.6 \%) \\
4(7.4 \%)\end{array}$ & $\begin{array}{l}43(76.8 \%) \\
13(23.2 \%)\end{array}$ & $\begin{array}{l}93(84.5 \%) \\
17(15.5 \%)\end{array}$ & $0.02^{* *}$ \\
\hline $\begin{array}{l}\text { Técnica quirúrgica, n (\%) } \\
\text { Mecánica } \\
\text { Femtosegundo }\end{array}$ & $\begin{array}{l}41(75.9 \%) \\
13(24.1 \%)\end{array}$ & $\begin{array}{l}38(67.9 \%) \\
18(32.1 \%)\end{array}$ & $\begin{array}{l}79(71.8 \%) \\
31(28.2 \%)\end{array}$ & $0.35^{* *}$ \\
\hline
\end{tabular}

que únicamente el estado refractivo previo tuvo una asociación estadísticamente significativa con la ganancia de líneas (OR: 3.78; IC 95\%: 1.15-12.45) (Tabla 3), y se encontró mayor cantidad de pacientes con astigmatismo miópico en el grupo de buen resultado visual en comparación con los del grupo control (Tabla 2).

No se reportó ninguna complicación intraoperatoria. En el postoperatorio solo se presentaron dos complicaciones (un paciente con extrusión 4 meses después de la cirugía y otro con desplazamiento de uno de los segmentos implantados luego de 3 meses), ambas en el grupo de controles, lo que representa una tasa de complicaciones del $1.81 \%$.

\section{Discusión}

El implante de segmentos intraestromales produce cambios significativos en los radios anterior y posterior de curvatura en los $3 \mathrm{~mm}$ centrales, lo que reduce el encurvamiento corneal y disminuye el astigmatismo irregular, con lo que mejora la $\mathrm{AV}$, sin cambios significativos en el grosor corneal ${ }^{8}$.

La recuperación visual luego de esta cirugía puede tomar de 3 a 12 meses, por lo que nuestra evaluación postoperatoria fue al menos al tercer mes de la cirugía, para tener cierta estabilidad en los resultados. Los criterios de selección deben ser rigurosos e incluyen la reducción grave de la $\mathrm{AV}$, córneas centrales claras con grosor central mínimo de 400 um y la falta de
Tabla 3. Análisis de asociación de los factores a mejoría visual tras implantación de segmentos intraestromales en pacientes con queratocono

\begin{tabular}{|l|c|c|}
\hline Factor de estudio & OR (IC 95\%) & P \\
\hline AVSC PRE & $2.76(0.79-9.70)$ & 0.112 \\
\hline AVCC PRE & $1.14(0.31-4.14)$ & 0.841 \\
\hline Grado de queratocono (I y II) & $0.62(0.29-1.39)$ & 0.23 \\
\hline Paquimetría & $0.99(0.98-1.00)$ & 0.458 \\
\hline Líneas que ganaba pre & $1.15(0.98-1.36)$ & 0.09 \\
\hline Astigmatismo miópico & $3.78(1.15-12.45)$ & 0.029 \\
\hline
\end{tabular}

AVCC PRE: agudeza visual con corrección preoperatoria; AVSC PRE: agudeza visual sin corrección preoperatoria; IC 95\%: intervalo de confianza al 95\%; OR: odds ratio.

visión funcional con correctores o lentes de contacto ${ }^{1}$. Existen varios nomogramas para la selección del segmento a implantar, basados en la refracción subjetiva preoperatoria, queratometría, topografía y datos de localización del cono, y se han reportado buenos resultados visuales y refractivos de ganancia de líneas de visión no corregida luego del implante'. Sin embargo, las sorpresas refractivas postoperatorias no son raras en términos de ganancia visual, por lo que no hay una predictibilidad establecida $^{9-13}$.

Los grupos estudiados fueron homogéneos en cuanto a edad y sexo. La mayor cantidad de pacientes operados en el instituto procedían de Lima (aproximadamente 
el $50 \%$ ) y un grupo importante $(37.4 \%)$ de zonas de altura. Algunos estudios indican que existen diferencias en el grosor central de la córnea según la altura de la región en la que viven, y es más delgada en pacientes que provienen de zonas a más de 2,500 m s.n.m. ${ }^{14,15}$. En nuestro estudio pudo haber un subregistro en este dato, ya que muchos pacientes indican como procedencia la capital y no su lugar de origen, ya que se trasladan a ella a recibir el tratamiento específico, por lo que este hallazgo no pudo ser estudiado adecuadamente.

La AV preoperatoria es un factor ampliamente estudiado por su estrecha relación con el resultado visual después de la implantación de segmentos intraestromales. Guyot, et al. encontraron que una AV mejor corregida preoperatoria de 0.30 mejor es un factor pronóstico para mejorar al menos 2 líneas de visión con correctores postcirugía ${ }^{16}$. Peña García, et al. reportaron que la AV a distancia corregida preoperatoria en promedio era mejor en el grupo de pacientes con mejoría visual postoperatoria en comparación a la del grupo que perdió líneas de visión tras la implantación de los segmentos, lo que encuentra una mayor efectividad de la cirugía en pacientes con AV a distancia corregida preoperatoria entre 0.3 a $0.4^{17}$. En un estudio más reciente, Sedaghat, et al. describieron como factor predictor de éxito la existencia de un valor alto de la resta entre la AV a distancia no corregida y la corregida preoperatorias, es decir, a mayor ganancia de líneas de visión preoperatoria con correctores, más oportunidad de éxito postoperatorio ${ }^{18}$. Estos resultados difieren de lo encontrado en nuestro estudio, en el cual los valores de la A sin corrección, la AV mejor corregida y la ganancia preoperatoria de líneas con correctores no tuvieron una asociación estadísticamente significativa con un buen resultado visual. Esta diferencia podría deberse a que, en los estudios mencionados, el éxito visual fue definido como la ganancia de 2 líneas o más de visión con correctores, mientras que en nuestro estudio fue definido como la ganancia de 3 líneas 0 más de visión sin correctores, lo que hace que la predictibilidad sea más difícil de establecer a este nivel.

Se encontró mayor número de ojos con queratometrías medias entre 48 a $52 \mathrm{D}$ (grado II), es decir, pacientes con estadios menos avanzados en el grupo de casos; sin embargo, no se pudo establecer una diferencia estadísticamente significativa para este factor. Alfonso, et al. mostraron que los pacientes con grados I y II mejoraban su AV luego de la cirugía con y sin correctores, a diferencia de los pacientes con grado III, en los cuales no había variación significativa de estos valores ${ }^{19}$. Este último hallazgo coincide con lo obtenido en nuestro estudio, en el cual el resultado visual final no se asoció con el grado de queratocono según queratometría y paquimetría previas a la cirugía. En contraste, Sedaghat, et al. no encontraron asociación de los valores de curvatura y la paquimetría preoperatorios con el éxito visual postoperatorio ${ }^{18}$. Esta diferencia se podría atribuir a que en nuestro estudio hubo un menor número de pacientes con queratocono de grado avanzado (> $53 \mathrm{D}$ ) en relación con los que tuvieron una queratometría media menor a $53 \mathrm{D}$.

El estado refractivo previo fue el único factor con asociación estadísticamente significativa al buen resultado visual postoperatorio. A su vez, hubo mayor cantidad de pacientes con astigmatismo simple o hipermetrópico en el grupo de los que no tuvieron un buen resultado visual. Algunos estudios muestran disminución de la magnitud del astigmatismo luego de la cirugía, como el de Piñero, et al., que reportó que este valor disminuyó de 0.75 a $2.88 \mathrm{D}$, lo que mejoró la AV corregida y no corregida postoperatoria de los pacientes, aunque con una tendencia a la hipocorrección ${ }^{20}$. Alfonso, et al. mostraron que en los pacientes con grados de queratocono I y II hubo una disminución importante del astigmatismo, lo que significó que la mayoría de ellos presentara mejoría importante en la visión postoperatoria con y sin correctores, en contraste con los que tenían grados más avanzados (III), en los que el cambio en el astigmatismo y la mejoría en la AV no corregida fueron mínimos ${ }^{19}$. Amanzadeh, et al. reportaron que la cirugía de implante aplana la córnea y mejora su irregularidad, lo que reduce la severidad del queratocono, pero esto es independiente de los cambios que se puedan presentar en la $\mathrm{AV}^{21}$. En nuestro estudio, encontramos una asociación estadísticamente significativa $(p<0.05)$ entre el astigmatismo miópico preoperatorio y un buen resultado visual posquirúrgico en comparación con los ojos de pacientes que tenían astigmatismo simple o hipermetrópicos. Este resultado es esperable teniendo en cuenta la acción de los segmentos intracorneales, que producen un aplanamiento corneal central, con lo que reducen el componente esférico miópico ${ }^{22}$.

La frecuencia de complicaciones postoperatorias reportadas es del 0.5 al $30 \%$. Entre las posibles complicaciones tenemos la neovascularización corneal, queratitis, depósitos alrededor del segmento del anillo, visión de halos, dolor, edema corneal, fotofobia y extrusión de segmentos, las cuales se puede presentar de días a años después de la colocación del implante $1,23,24$.

En nuestro estudio, solo se reportaron complicaciones en dos pacientes, lo que representó una tasa de 
complicaciones postoperatorias del $1.81 \%$, la cual está dentro de lo reportado en la literatura médica.

La inserción de segmentos se puede realizar mecánicamente 0 asistida con láser de femtosegundo. Se han reportado buenos resultados visuales con la técnica mecánica, con un porcentaje aceptable de complicaciones según la gravedad de la condición ectásica preexistente ${ }^{25}$. El láser femtosegundo, por otro lado, reduce las complicaciones debido a la rapidez, precisión, facilidad de creación del canal y un centrado asociado con un edema estromal mínimo, lo que permite que el procedimiento de implantación del anillo corneal sea más rápido, fácil y seguro, con una profundidad de implantación precisa ${ }^{26,27}$. Actualmente existen reportes de mejoría de la AV con ambas técnicas sin diferencias estadísticamente significativas entre los resultados visuales y refractivos ${ }^{28}$. En nuestro estudio no encontramos asociación entre la técnica operatoria utilizada para la implantación de los segmentos intraestromales y el resultado visual postoperatorio. Esto es concordante con Kubaloglu, et al. y Piñero, et al., quienes evidenciaron valores similares de aplanamiento corneal, disminución del equivalente esférico y mejoría de la AV sin corrección con ambas técnicas, además de tasas similares de complicaciones al comparar el tipo de técnica utilizada ${ }^{8,29}$.

Una limitación de nuestro estudio fue la cantidad de participantes, un mayor número de pacientes, principalmente con queratoconos avanzados, podría haber modificado la asociación de la queratometría media previa y el resultado visual postoperatorio. Asimismo, se evaluaron los resultados al menos 3 meses después de la cirugía, pero el tiempo del seguimiento postoperatorio no fue el mismo para todos los pacientes. Además, a diferencia de otros estudios que definen el éxito visual postoperatorio como la mejoría de líneas de visión con correctores, definimos el buen resultado visual como la ganancia de tres líneas o más sin correctores, ya que consideramos que es la visión sin correctores la que tiene mayor impacto en el paciente. Si bien la evidencia muestra que los resultados visuales de esta operación son poco predecibles ${ }^{12}$, esta poca predictibilidad puede ser aún mayor cuando se busca una mejoría visual sin corrección.

\section{Conclusiones}

De los factores estudiados (AV, refracción, paquimetría, grado de queratocono y técnica operatoria), solo el estado refractivo previo presentó asociación con el buen resultado visual postoperatorio sin correctores. La presencia de complicaciones postoperatorias fue baja $(1.81 \%)$.

\section{Conflicto de intereses}

Los autores declaran no tener intereses financieros en la realización de este estudio.

\section{Financiamiento}

Los autores declaran que esta investigación y artículo fue autofinanciado.

\section{Responsabilidades éticas}

Protección de personas y animales. Los autores declaran que para esta investigación no se han realizado experimentos en seres humanos ni en animales.

Confidencialidad de los datos. Los autores declaran que han seguido los protocolos de su centro de trabajo sobre la publicación de datos de pacientes.

Derecho a la privacidad y consentimiento informado. Los autores declaran que en este artículo no aparecen datos de pacientes.

\section{Bibliografía}

1. Mohammadpour M, Heidari Z, Hashemi H. Updates on Managements for Keratoconus. J Curr Ophthalmol. 2018;30(2):110-24

2. Israel M, Yousif MO, Osman NA, Nashed M, Abdelfattah NS. Keratoconus correction using a new model of intrastromal corneal ring segments. J Cataract Refract Surg. 2016:42(3):444-54.

3. Blériot $A$, Martin $E$, Lebranchu $P$, Zimmerman $K$, Libeau L, Weber M, et al. Comparison of 12-month anatomic and functional results between Z6 femtosecond laser-assisted and manual trephination in deep anterior lamellar keratoplasty for advanced keratoconus. J Fr Ophtalmol. 2017; 40(6):e193-200.

4. Wahba SS, Roshdy MM, Fikry RR, Abdellatif MK, Abodarahim AM. Topographic Asymmetry Indices: Correlation between Inferior Minus Superior Value and Index of Height Decentration. J Ophthalmol. 2018;2018:1-4.

5. Shajari M, Jaffary I, Herrmann K, Grunwald C, Steinwender G, Mayer WJ, et al. Early Tomographic Changes in the Eyes of Patients With Keratoconus. J Refract Surg. 2018;34(4):254-9.

6. Arantes JCD, Coscarelli S, Ferrara P, Araújo LPN, Ávila M, Torquetti L. Intrastromal Corneal Ring Segments for Astigmatism Correction after Deep Anterior Lamellar Keratoplasty. J Ophthalmol. 2017; 2017:8689017.

7. Naderan M, Jahanrad A, Balali S. Histopathologic findings of keratoconus corneas underwent penetrating keratoplasty according to topographic measurements and keratoconus severity. Int J Ophthalmol. 2017; 10(11):1640-6.

8. Sedaghat M-R, Momeni-Moghaddam H, Belin MW, Zarei-Ghanavati S, Akbarzadeh R, Sabzi F, et al. Changes in the ABCD Keratoconus Grade After Intracorneal Ring Segment Implantation. Cornea. 2018; 37(11):1431-7.

9. Lyra D, Ribeiro G, Torquetti L, Ferrara P, Machado A, Lyra JM. Computational Models for Optimization of the Intrastromal Corneal Ring Choice in Patients With Keratoconus Using Corneal Tomography Data. J Refract Surg. 2018:34(8):547-50.

10. Utine CA, Ayhan Z, Durmaz Engin C. Effect of intracorneal ring segment implantation on corneal asphericity. Int J Ophthalmol. 2018;11(8):1303-7.

11. Muftuoglu O, Aydin R, Kilic Muftuoglu I. Persistence of the Cone on the Posterior Corneal Surface Affecting Corneal Aberration Changes After Intracorneal Ring Segment Implantation in Patients With Keratoconus. Cornea. 2018;37(3):347-53. 
12. Ferrara G, Torquetti L, Ferrara P, Merayo-Lloves J. Intrastromal corneal ring segments: visual outcomes from a large case series. Clin Experiment Ophthalmol. 2012;40(5):433-9.

13. Zadnik K, Money S, Lindsley K. Intrastromal corneal ring segments for treating keratoconus. Cochrane Database Syst Rev. 2019; 14;5(5): CD011150.

14. Patyal S, Arora A, Yadav A, Sharma VK. Corneal Thickness in Highlanders. High Alt Med Biol. 2017;18(1):56-60.

15. Liu HM, Bai CH, Liou CM, Chiou HY, Chen C. Central Corneal Thickness of Healthy Lowlanders at High Altitude: A Systematic Review and Meta-Analysis. Curr Eye Res. 2018;43(4):460-5.

16. Guyot C, Libeau L, Vabres B, Weber M, Lebranchu P, Orignac I. Refractive outcome and prognostic factors for success of intracorneal ring segment implantation in keratoconus: A retrospective study of 75 eyes. J Fr Ophtalmol. 2018;42(2):118-26.

17. Peña-García P, Vega-Estrada A, Barraquer RI, Burguera-Giménez N, Alio JL. Intracorneal ring segment in keratoconus: A model to predic visual changes induced by the surgery. Investig Ophthalmol Vis Sci. 2012;53(13):8447-57.

18. Sedaghat MR, Momeni-Moghaddam H, Piñero DP, Akbarzadeh R, Moshirfar M, Bamdad S, et al. Predictors of Successful Outcome following Intrastromal Corneal Ring Segments Implantation. Curr Eye Res. 2019;44(7):707-15.

19. Alfonso JF, Lisa C, Fernández-Vega L, Madrid-Costa D, Montés-Micó R. Intrastromal corneal ring segment implantation in 219 keratoconic eyes at different stages. Graefe's Arch Clin Exp Ophthalmol. 2011;249(11): 1705-12.

20. Piñero DP, Alió JL, Teus MA, Barraquer RI, Michael R, Jiménez R. Modification and refinement of astigmatism in keratoconic eyes with intrastromal corneal ring segments. J Cataract Refract Surg. 2010;36(9):1562-72.
21. Amanzadeh $\mathrm{K}$, Elham $\mathrm{R}$, Jafarzadepur $\mathrm{E}$. Effects of single-segment Intacs implantation on visual acuity and corneal topographic indices of keratoconus. J Curr Ophthalmol. 2017;29(3):189-93.

22. Söğütlü $E$, Piñero $D P$, Kubaloglu $A$, Alio JL, Cinar $Y$. Elevation Changes of Central Posterior Corneal Surface After Intracorneal Ring Segment Implantation in Keratoconus. Cornea. 2012;31(4):387-95.

23. Oatts JT, Savar L, Hwang DG. Late extrusion of intrastromal corneal ring segments: A report of two cases. Am J Ophthalmol Case Reports. 2017:8:67-70.

24. Piñero DP, Alio JL. Intracorneal ring segments in ectatic corneal disease - a review. Clin Experiment Ophthalmol. 2010;38(2):154-67.

25. Flecha-Lescún J, Calvo B, Zurita J, Ariza-Gracia MÁ. Template-based methodology for the simulation of intracorneal segment ring implantation in human corneas. Biomech Model Mechanobiol. 2018;17(4):923-38.

26. Mounir A, Radwan G, Mohamed Farouk M, Mohamed Mostafa E. Femtosecond-assisted intracorneal ring segment complications in keratoconus: from novelty to expertise. Clin Ophthalmol. 2018;12(4):957-64.

27. Monteiro T, Alfonso JF, Franqueira N, Faria-Correia F, Ambrósio R, Madrid-Costa D. Predictability of Tunnel Depth for Intrastromal Corneal Ring Segments Implantation Between Manual and Femtosecond Laser Techniques. J Refract Surg. 2018;34(3):188-94.

28. Kubaloglu A, Sari ES, Cinar Y, Cingu K, Koytak A, Coskun E, et al. Comparison of mechanical and femtosecond laser tunnel creation for intrastromal corneal ring segment implantation in keratoconus: prospective randomized clinical trial. J Cataract Refract Surg. 2010;36(9): 1556-61.

29. Piñero DP, Alio JL, El Kady B, Coskunseven E, Morbelli H, Uceda-Montanes A, et al. Refractive and Aberrometric Outcomes of Intracorneal Ring Segments for Keratoconus: Mechanical versus Femtosecond-assisted Procedures. Ophthalmology. 2009;116(9):1675-87. 